\title{
Optimal diagonal-norm SBP operators
}

\author{
Ken Mattsson ${ }^{* 1}$, Martin Almquist ${ }^{\dagger 1}$ and Mark H. Carpenter ${ }^{\ddagger 2}$ \\ ${ }^{1}$ Department of Information Technology, Uppsala University \\ ${ }^{2}$ Computational Aerosciences Branch, NASA Langley Research \\ Center, Hampton, VA 23681-2199. Work performed while \\ visiting the Department of Information Technology, Uppsala \\ University.
}

September 3, 2013

\begin{abstract}
Optimal boundary closures are derived for first derivative, finite difference operators of order 2, 4,6 and 8. The closures are based on a diagonal-norm summation-by-parts (SBP) framework, thereby guaranteeing linear stability on piecewise curvilinear multi-block grids and entropy stability for nonlinear equations that support a convex extension. The new closures are developed by enriching conventional approaches with additional boundary closure stencils and non-equidistant grid distributions at the domain boundaries. Greatly improved accuracy is achieved near the boundaries, as compared with traditional diagonal norm operators of the same order. The superior accuracy of the new optimal diagonal-norm SBP operators is demonstrated for linear hyperbolic systems in one dimension and for the nonlinear compressible Euler equations in two dimensions.
\end{abstract}

Key words: finite difference methods, multi-block grids, high order accuracy, stability, boundary treatment, Euler equations

\footnotetext{
*ken.mattsson@it.uu.se

${ }^{\dagger}$ martin.almquist@it.uu.se

${ }^{\ddagger}$ mark.h.carpenter@nasa.gov
} 


\section{Introduction}

Wave-propagation problems frequently require farfield boundaries to be positioned many wavelengths away from the disturbance source. Efficient simulation of these problems requires numerical techniques capable of accurately propagating disturbances over long distances. It is well know that high-order finite difference methods (HOFDMs) are ideally suited for problems of this type. (See the pioneering paper by Kreiss and Oliger [22].) Not all high-order spatial operators are applicable, however. For example, schemes that are GK-S stable [13], while being convergent to the true solution as $\Delta x \rightarrow 0$, may experience nonphysical solution growth in time [5], thereby limiting their efficiency for longtime simulations. Thus, it is imperative to use HOFDMs that do not allow nonphysical solution growth in time - a property termed "strict stability" [12]. Deriving a strictly stable, accurate, and conservative HOFDM is a significant challenge that has received considerable past attention. (For examples, see $[24,41,38,1,3,15,39,14]$.)

A robust and well-proven high-order finite difference methodology that ensures the strict stability of time-dependent partial differential equations (PDEs) is the summation-by-parts-simultaneous approximation term (SBPSAT) method. The SBP-SAT method combines semi-discrete operators that satisfy a summation-by-parts (SBP) formula [20], with physical boundary conditions implemented using the simultaneous approximation term (SAT) method [5]. Examples of the SBP-SAT approach can be found in [33, 34, 35, 28, 30, 31, 36, 27, 43, 23, 9, 29, 17, 16, 19].

An added benefit of the SBP-SAT method is that it naturally extends to multi-block geometries while retaining the essential single-block properties: strict stability, accuracy, and conservation [6]. Thus, problems involving complex domains or non-smooth geometries are easily amenable to the approach. References [27, 30, 18] report applications of the SBP-SAT method to problems involving nontrivial geometries.

A wide variety of numerical methods may be expressed as summation-byparts operators; examples include finite-difference operators, spectral collocation $[8,7]$, and some finite-volume methods [45]. Herein we focus exclusively on finite difference operators. Finite-difference operators may be further categorized by the structure of their norm: a) diagonal, b) diagonal interior with block boundary closures, c) fully banded. The early papers [21, 40] report first derivative SBP operators up to 6 th order, based on diagonal-norms and block-norms, while in [4] a 4th order accurate first derivative Padé type banded SBP operator is derived. The SBP framework may also be used to develop second-derivative operators. In [28] diagonal- and block-norm SBP operators up to 8 th order were derived for both the first and second deriva- 
tives.

Matrix structural constraints required by all SBP operators preclude uniform spatial accuracy for diagonal norm finite-difference operators [21]. Indeed, a 2pth order diagonal-norm SBP operator may be closed at the boundaries with at most pth order accurate biased stencils. For first order hyperbolic problems, this implies a suboptimal convergence rate (i.e., global convergence) of $(p+1)$ th order when using a $2 p t h$ order interior operator. By comparision, a block norm SBP operator with an identical interior stencil may be closed at the boundaries with $(2 p-1)$ th order accurate stencils, thereby yielding optimal $2 p t h$ order convergence. Similarly, strongly parabolic problems and second order hyperbolic problems convergence at a rate of $(p+2)$ th order using a $2 p t h$ order diagonal-norm SBP operator. (See $[11,46]$ for more information on the accuracy of finite difference approximations.)

At first glance, it would appear that block norm or banded SBP operators would be preferred for simulations with stringent accuracy requirements. It is well known, however, that block-norm SBP operators may exhibit instabilities when applied to problems mapped onto curvilinear grids [42], while diagonal norm operators remain stable. The same instability mechanism exists on Cartesian grids for second order systems with variable coefficients [32].

Furthermore, recent developments appearing in reference [10] provide a general procedure for developing entropy consistent and entropy stable SBP schemes for systems of equations that support a convex extension (e.g., the entropy in the compressible Navier-Stokes equations). This procedure is only valid, however, for diagonal norm SBP spatial operators. For these reasons diagonal-norm operators have gained more interest in practical applications.

State-of-the-art diagonal norm finite-difference operators approach a point of dimenishing return with 8 th order interior operators and 4 th order boundary closures $[28,30]$, thereby achieving 5 th order convergence (for first order hyperbolic systems and incompletely parabolic systems) on curvilinear grids. An optimized 10th order diagonal-norm SBP operator appears in [9], but exhibits large negative eigenvalues that separate from the cluster, leading to very small time-steps for explicit time-stepping methods.

Herein, diagonal-norm SBP finite difference operators are derived with highly optimized boundary closures. Although the resulting operators are formally $5 t h$-order accurate (for the $8 t h$ order case), the leading order error constants are reduced by several orders of magnitude. Thus, very accurate simulations can be performed on relatively coarse meshes. The optimized boundary operators utilize additional unknowns in the SBP boundary closures, as well as a non-equidistant point distribution at the first three bound- 
ary points in the domain. The superior accuracy properties of the newly derived diagonal-norm SBP operators are demonstrated for linear hyperbolic problems in 1-D and the compressible Euler equations in 2-D, where the computational domain consists of two curvilinear grids.

In Section 2 the SBP-SAT method is introduced in one spatial dimension. In Section 3 the accuracy and stability properties of the newly developed SBP operators are verified by performing 1-D numerical simulations. Verification of accuracy and stability by numerical studies of the 2-D problem is performed in Section 4. Section 5 summarizes the work. The SBP operators are presented in Appendix.

\section{The finite difference method}

The focus in the present study is the derivation of accuracy-optimized diagonalnorm SBP operators. It is well known that the traditional diagonal-norm SBP operators suffer from the low accuracy at the boundaries. It is further shown [21] that 10th order diagonal-norm SBP operator require eleven boundary stencils to achieve optimal accuracy. In a previous paper [25] we derived a 10th order diagonal SBP operator using eleven boundary points. The accuracy is still very low compared to the internal scheme, and the operator is not attractive.

\subsection{Definitions}

The following definitions are needed later in the present study. Let $\mathbf{u}, \mathbf{v} \in$ $L^{2}[0,1]$ where $\mathbf{u}^{T}=\left[u^{(1)},, u^{(2)}, \ldots, u^{(k)}\right]$ and $\mathbf{v}^{T}=\left[v^{(1)},, v^{(2)}, \ldots, v^{(k)}\right]$ are real-valued vector functions with $k$ components. Let the inner product be defined by $(\mathbf{u}, \mathbf{v})=\int_{0}^{1} \mathbf{u}^{T} \mathbf{A}(x) \mathbf{v} d x, \mathbf{A}(x)=\mathbf{A}^{T}(x)>0$, and let the corresponding norm be $\|\mathbf{u}\|_{A}^{2}=(\mathbf{u}, \mathbf{u})$.

The domain $(0 \leq x \leq 1)$ is discretized using $m$ grid points:

$$
\mathbf{x}=\left[x_{1}, x_{2}, \ldots, x_{m-1}, x_{m}\right]^{T},
$$

i.e., $\mathbf{x}$ denotes a vector holding the grid-points. For an equidistant grid we have

$$
x_{i}=(i-1) h, \quad i=1,2, \ldots, m, \quad h=\frac{1}{m-1} .
$$

In the present study we will allow the 3 grid-points closest to the boundary to be non-equidistant to improve accuracy.

The approximate solution vector is given by $v^{T}=\left[v^{(1)}, v^{(2)}, \ldots, v^{(k)}\right]$, where $v^{(j)}=\left[v_{1}^{(j)}, v_{2}^{(j)}, \ldots, v_{m}^{(j)}\right]$ is the discrete solution vector of the $j t h$ component. Similarly, we define an inner product for discrete real-valued vector 
functions $u, v \in \mathbf{R}^{\mathbf{k} \times \mathbf{m}}$ by $(u, v)_{H A}=u^{T} A \tilde{H} v$, where $\tilde{H}=I_{k} \otimes H$ is positive definite ( $H$ is a symmetric positive definite $m \times m$ matrix) and $A$ is the projection of $\mathbf{A}(x)$ onto the block diagonals. If $\mathbf{A}(x)$ is a diagonal $k \times k$ matrix, $A$ is a diagonal $(\mathrm{km}) \times(\mathrm{km})$ matrix. $I_{k}$ denotes the unit matrix of size $k \times k$. The corresponding norm is $\|v\|_{H A}^{2}=v^{T} \tilde{H} A v$.

Remark The matrix product $\tilde{H} A$ defines a norm if and only if $\tilde{H} A$ is positive definite. For variable $A(x)$ this can only be guaranteed if the norm $H$ is a diagonal matrix (see [42] for a detailed study on this). Variable coefficients are often present due to the underlying physics or the geometry requiring the introduction of curvilinear grids. We will focus on both these issues in the present study.

Here we make use of the Kronecker product:

$$
C \otimes D=\left[\begin{array}{ccc}
c_{0,0} D & \cdots & c_{0, q-1} D \\
\vdots & & \vdots \\
c_{p-1,0} D & \cdots & c_{p-1, q-1} D
\end{array}\right],
$$

where $C$ is a $p \times q$ matrix and $D$ is an $m \times n$ matrix. Two useful rules for the Kronecker product are $(A \otimes B)(C \otimes D)=(A C) \otimes(B D)$ and $(A \otimes B)^{T}=$ $A^{T} \otimes B^{T}$. The following vectors will be frequently used:

$$
e_{1}=[1,0, \ldots, 0]^{T}, \quad e_{m}=[0, \ldots, 0,1]^{T} .
$$

To define the SBP-SAT method, we present the following definition (first stated in $[31,27])$ :

Definition 2.1 An explicit 2pth-order accurate finite difference scheme with minimal stencil width for the Cauchy problem is denoted a 2pth-order accurate narrow-stencil.

The SBP definitions can be found in earlier papers (see for example [28, $31,27,26,29,32])$. For completeness we restate them below for the first derivative:

Definition 2.2 A difference operator $D_{1}=H^{-1}\left(Q-\frac{1}{2} e_{1} e_{1}^{T}+\frac{1}{2} e_{m} e_{m}^{T}\right)$, approximating $\partial / \partial x$, using a 2 pth-order accurate narrow-stencil, is said to be a 2pth-order first-derivative SBP operator if $H$ is symmetric and positive definite, and $Q+Q^{T}=0$.

Definition 2.3 A difference operator $D_{1}=H^{-1}\left(Q-\frac{1}{2} e_{1} e_{1}^{T}+\frac{1}{2} e_{m} e_{m}^{T}\right)$, approximating $\partial / \partial x$, using a 2 pth-order accurate narrow-stencil, is said to be a 2pth-order diagonal-norm first-derivative SBP operator if $H$ is diagonal and positive definite, and $Q+Q^{T}=0$. 


\subsection{Optimal diagonal-norm SBP operators}

In the present paper newly derived diagonal-norm SBP operators are presented that are optimal in the sense that we have used the "free" parameters always present when constructing SBP boundary closures (present in $Q$ and $H$ at the boundaries) to optimize the leading truncation errors for the boundary stencils.

The following new definition is central in the present study.

Definition 2.4 Let $\mathbf{x}^{q}$ be the projection of the polynomial $\frac{x^{q}}{q !}$ onto the discrete grid-points, i.e., a vector denoted $\mathbf{x}$.

Let $e_{(q)}=H \mathbf{x}^{q}-\left(Q-\frac{1}{2} e_{1} e_{1}^{T}+\frac{1}{2} e_{m} e_{m}^{T}\right) \mathbf{x}^{q-1}$ be the qth order error vector. We say that $D_{1}=H^{-1}\left(Q-\frac{1}{2} e_{1} e_{1}^{T}+\frac{1}{2} e_{m} e_{m}^{T}\right)$ is $2 p t h$-order if $e_{(q)}$ vanish for $q=1 \ldots 2 p$, in the interior and for $q=1 \ldots p$, at the boundaries.

The traditional $2 p t h$ order diagonal-norm SBP operators are defined on an equidistant grid, with $2 p$ number of boundary stencils that are only $p t h$ order accurate. The 6 th order operator has one "free" parameter in $Q$, while the 8th order one has three. By choosing the "free" parameters in $H$ and $Q$ to minimize the norm of the leading error vector, i.e., $e_{(p+1)}$ for the $2 p t h$ order case, we obtain the most accurate SBP operators possible on equidistant grids. (This procedure leads to linear equations to solve.) One way to further reduce the leading truncation errors (that are $p t h$ order) at the boundaries is to introduce more boundary stencils. This does however only work up to the $6 t h$ order case on an equidistant grid, since a valid SBP solution to this minimization can not be obtained using more than 8 boundary points. If there are free parameters left after the first minimization, we continue to minimize the norm of the next leading error vector, i.e., $e_{(p+2)}$. In the present study we have derived optimal $2 n d, 4 t h$ and $6 t h$ order accurate diagonal-norm SBP operators where we have used $2 p+2$ boundary stencils on an equidistant grid, presented in Appendix.

A way to further reduce the truncation errors and at the same time allow for a much more accurate 8th order diagonal-norm SBP operator is to allow for a non-equidistant grid at the boundaries. Hence, we allow the location of the first boundary points to be "free" in the minimization. Allowing for a "free" location of the boundary points, however, leads to a non-linear minimization, and by using MAPLE we have found that no more than three "free" boundary points can be allowed (it simply becomes too computationally demanding). Let $\mathbf{x} \in[0,1]$. Introduce $d=d_{1}+d_{2}+d_{3}$, where $h d_{1}$ is the distance between the first and second grid-point, $h d_{2}$ is the distance between the second and third grid-point, and $h d_{3}$ is the distance between the third and fourth grid-point. Here $h=1 /(2 d+(m-7))$ and $m$ is the number of 
grid-points. The grid-points are located at

$$
\begin{gathered}
\mathbf{x}=\left[0, d_{1} h,\left(d_{1}+d_{2}\right) h, d h,(d+1) h,(d+2) h, \ldots,\right. \\
\left.(d+(m-7)) h, 1-d h, 1-\left(d_{1}+d_{2}\right) h, 1-d_{1} h, 1\right],
\end{gathered}
$$

where $d_{1}, d_{2}$ and $d_{3}$ are unknowns in the minimization.

We have derived $2 n d, 4 t h, 6 t h$ and $8 t h$ order accurate diagonal SBP operators, where the location of the first three grid-points (closest to the boundary) are non-equidistant, see Figure 1. For the $2 n d$ order case we use three boundary stencils, and for the $4 t h$ and 6 th order cases we use 5 and 7 boundary stencils, respectively. For the $8 t h$ order case we are restricted to 8th boundary stencils. In the present study these new diagonal-norm SBP operators will be referred to as optimal. All operators are presented in Appendix. The results presented in Sections 3 and 4 show that there is a huge gain in accuracy by allowing the grid-points to be non-equidistant at the boundaries.

Remark The process of deriving optimal SBP operators yield the optimal positions for the grid-points, resulting in a non-equidistant grid close to the boundaries. The gain in efficiency is rather high, especially for the $8 t h$ order accurate case.

\subsection{The SBP-SAT method}

Consider

$$
\mathbf{u}_{t}=\mathbf{A} \mathbf{u}_{x}, \quad 0 \leq x \leq 1, \quad t \geq 0,
$$

where $\mathbf{A}=\mathbf{A}^{T}$ is a constant coefficient matrix. (We also assume initial data $\mathbf{u}(x, 0)=\mathbf{f}(x)$.) This equation is now mapped onto a curvilinear grid $x=x(\xi)$,

$$
\mathbf{J} \mathbf{u}_{t}=\mathbf{A} \mathbf{u}_{\xi}, \quad 0 \leq \xi \leq 1, \quad t \geq 0,
$$

where $\mathbf{J}=\xi_{x}^{-1}$ is the Jacobian matrix.

We diagonalize $\mathbf{A}$ by a similar transformation, $\mathbf{A}=\mathbf{T}^{\mathbf{T}} \boldsymbol{\Lambda} \mathbf{T}$, where $\boldsymbol{\Lambda}$ holds the eigenvalues to $\mathbf{A}$. We will apply boundary conditions to the ingoing characteristics, to specify the correct number of boundary conditions. We introduce

$$
2 \mathbf{A}_{ \pm}=\mathbf{T}^{\mathbf{T}}(\boldsymbol{\Lambda} \pm|\boldsymbol{\Lambda}|) \mathbf{T} .
$$

At the left (L) and right (R) boundaries we specify data for $\mathbf{A}_{+} \mathbf{u}$ and $\mathbf{A}_{-} \mathbf{u}$ respectively, i.e,

$$
\mathbf{A}_{-} \mathbf{u}_{\mathbf{L}}=\mathbf{A}_{-} \mathbf{g}_{\mathbf{L}}, \quad \mathbf{A}_{+} \mathbf{u}_{\mathbf{R}}=\mathbf{A}_{+} \mathbf{g}_{\mathbf{R}}
$$




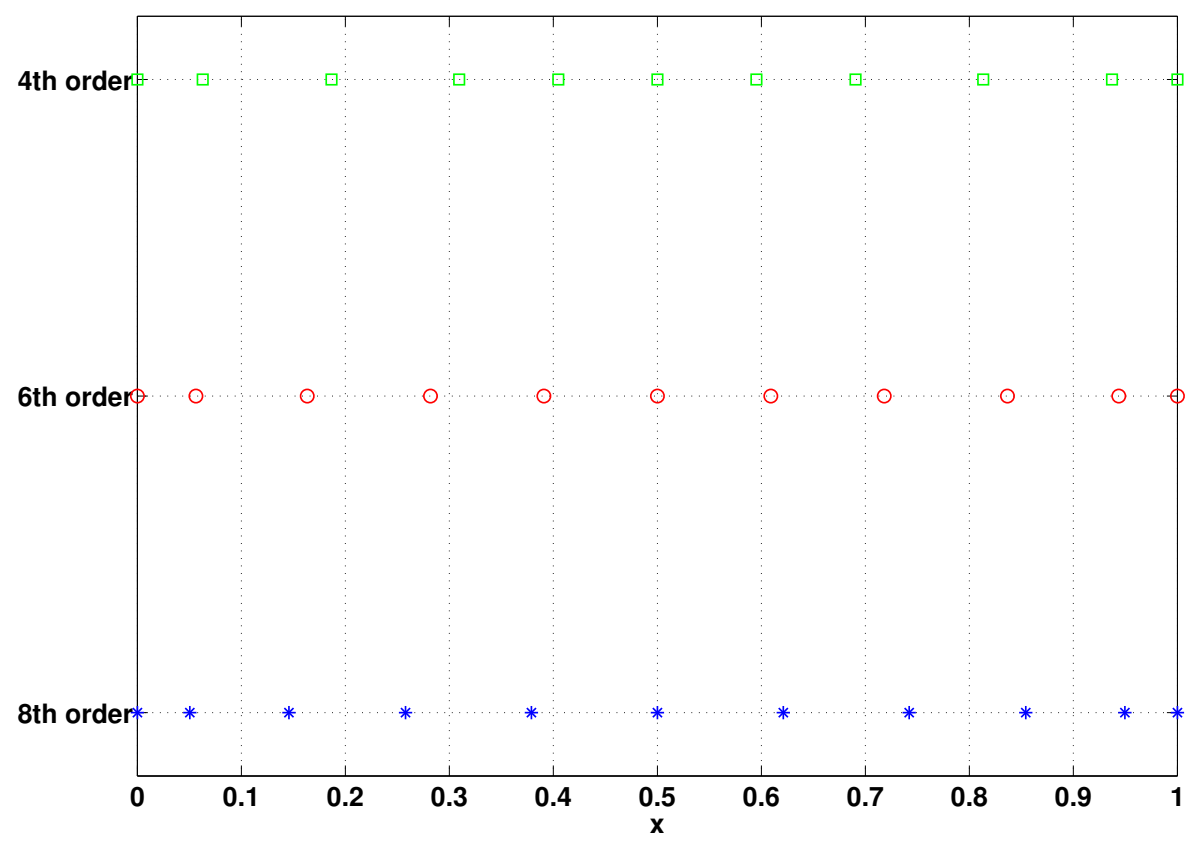

Figure 1: The location of the grid-points for the optimal 4th, 6th and 8th order accurate cases, using $m=11$. The location of the first three grid-points (closest to the boundary) are non-equidistant. A Cartesian grid would have the grid-points at the vertical dotted lines.

where $\mathbf{u}_{\mathbf{L}, \mathbf{R}}$ are the unknowns, and $\mathbf{g}_{\mathbf{L}, \mathbf{R}}$ the corresponding data at the Left and Right boundaries, respectively.

Multiplying (2) by $\mathbf{u}^{T}$, integrating by parts, adding the transpose and imposing the ingoing characteristics (4) leads to,

$$
\frac{d}{d t}\|\mathbf{u}\|_{J}^{2}=\mathbf{u}_{L}^{T} \mathbf{A}_{-} \mathbf{u}_{L}+\mathbf{g}_{L}^{T} \mathbf{A}_{+} \mathbf{g}_{L}-\mathbf{u}_{R}^{T} \mathbf{A}_{+} \mathbf{u}_{R}-\mathbf{g}_{R}^{T} \mathbf{A}_{-} \mathbf{g}_{R}
$$

Hence, the problem defined by (2) and (4) is strongly well posed [12].

The semi-discrete problem using the SBP-SAT method leads to

$$
\tilde{J} v_{t}=A \otimes D_{1}+A_{-} \otimes H^{-1} e_{1}\left(v_{L}-g_{L}\right)-A_{+} \otimes H^{-1} e_{m}\left(v_{R}-g_{R}\right),
$$

where $\tilde{J}=I_{k} \times J$ and $J$ is the projection of the Jacobian matrix onto the diagonal. $v_{L}=I_{k} \otimes e_{1}^{T} v$ and $v_{R}=I_{k} \otimes e_{m}^{T} v$ are the unknowns at the left and right boundaries.

We multiply (6) by $v^{T} \tilde{H}$ and add the transpose to obtain,

$$
v_{t}^{T} \tilde{H} \tilde{J} v+v^{T} \tilde{J} \tilde{H} v_{t}=B T_{L}+B T_{R},
$$


where

$$
\begin{aligned}
& B T_{L}=+v_{L}^{T} A_{-} v_{L}+g_{L}^{T} A_{+} g_{L}-\left(v_{L}-g_{L}\right)^{T} A_{+}\left(v_{L}-g_{L}\right), \\
& B T_{R}=-v_{R}^{T} A_{+} v_{R}-g_{R}^{T} A_{-} g_{R}+\left(v_{R}-g_{R}\right)^{T} A_{-}\left(v_{R}-g_{R}\right) .
\end{aligned}
$$

Obtaining an energy estimate requires that the following holds

$$
H J=J H,
$$

such that the LHS in (7) can be written as $v^{T} \tilde{H} \tilde{J} v_{t}+v_{t}^{T} \tilde{H} \tilde{J} v=\frac{d}{d t}\|v\|_{H J}^{2}$. Hence, stability on curvilinear grids can only be guaranteed if the norm $H$ is diagonal.

\section{Computations in 1-D}

\subsection{First order systems}

We study the hyperbolic system (2) where

$$
\mathbf{A}=\left[\begin{array}{ll}
0 & 1 \\
1 & 0
\end{array}\right]
$$

and where we specify the ingoing characteristics. Here

$$
2 \mathbf{A}_{+}=\left[\begin{array}{ll}
1 & 1 \\
1 & 1
\end{array}\right], \quad 2 \mathbf{A}_{-}=\left[\begin{array}{cc}
-1 & 1 \\
1 & -1
\end{array}\right] .
$$

In this section we will make use of the following coordinate transformation,

$$
x=x(\xi)=\xi\left(\frac{e^{-\left(\xi-\frac{4}{5}\right)^{2}}}{e^{-\left(1-\frac{4}{5}\right)^{2}}}\right)^{l}, \quad \xi \in[0,1]
$$

Here $l$ is a non-negative integer, where the case $l=0$ corresponds to a Cartesian grid. A larger value of $l$ leads to a denser clustering of grid-points close to the boundaries.

We approximate this system with the SBP-SAT method, given by (6). To test the accuracy of the newly developed diagonal-norm SBP operators we choose the analytic solution $\mathbf{u}=[\sin (n \pi x),-\sin (n \pi x)]^{T}$, where $n$ is an integer number. The convergence rate is calculated as

$$
q=\log _{10}\left(\frac{\left\|u-v^{\left(m_{1}\right)}\right\|_{h}}{\left\|u-v^{\left(m_{2}\right)}\right\|_{h}}\right) / \log _{10}\left(\frac{m_{1}}{m_{2}}\right)^{1 / d},
$$


where $d$ is the dimension ( $d=1$ in the 1 -D case), $u$ is the analytic solution, and $v^{\left(m_{1}\right)}$ the corresponding numerical solution with $m_{1}$ unknowns. $\| u-$ $v^{\left(m_{1}\right)} \|_{h}$ is the discrete $l_{2}$ norm of the error.

In Tables 1 - 3 we compare the result using the traditional and optimal diagonal-norm SBP operators, with no grid-stretching, i.e., $l=0$ in (11). The solution is integrated in time using the $4 t h$ order accurate Runge-Kutta method using a CFL of 0.5 (there is little difference between the time-step restrictions using the traditional and optimal SBP operators). In Figure 2 we plot the $l_{2}$-errors (taken from Tables 2 and 3 ) versus the number of grid-points for the traditional and optimal $6 t h$ and $8 t h$ order accurate SBP operators in a $\log -\log$ plot, to more clearly see the huge gain in efficiency going to optimal SBP operators.

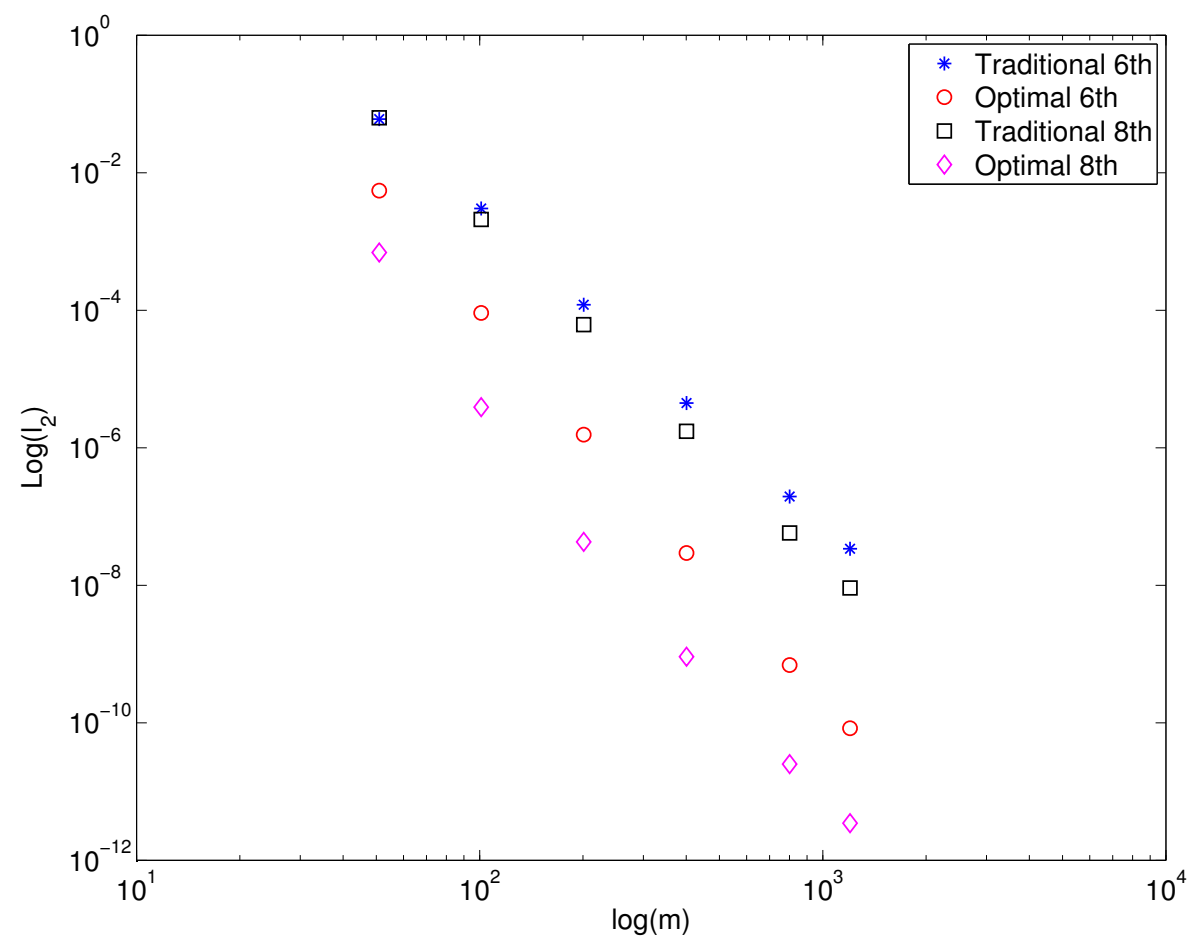

Figure 2: Comparing the traditional and optimal 6th and 8th order accurate SBP operators in a $\log -\log$ plot, results taken from Tables 2 and 3.

To validate the importance of using diagonal-norm SBP operators for this problem we present in Figure 3 the eigenvalues to (6) where the system is defined by (10), on a grid defined by (11) with $l=2$ and $m=51$. We compare the spectra using the 8th order optimal diagonal-norm SBP operator and the corresponding block-norm SBP operator. The largest real part of the spectrum is referred to as $\lambda_{\max }$. A non-positive value is required for stability. 


\begin{tabular}{lllllll}
\hline$N$ & $\log l_{2}$ & $q$ & $\log l_{2}$ & $q$ & $\log l_{2}$ & $q$ \\
\hline 51 & -1.09 & 0.00 & -1.11 & 0.00 & -1.25 & 0.00 \\
101 & -2.15 & 3.52 & -2.34 & 4.10 & -2.42 & 3.88 \\
201 & -3.12 & 3.23 & -3.56 & 4.04 & -3.61 & 3.95 \\
401 & -4.05 & 3.07 & -4.76 & 3.98 & -4.80 & 3.97 \\
801 & -4.96 & 3.02 & -5.94 & 3.92 & -6.00 & 3.98 \\
1201 & -5.49 & 3.01 & -6.61 & 3.81 & -6.70 & 3.97 \\
\hline
\end{tabular}

Table 1: $\log \left(l_{2}-\right.$ errors $)$ and convergence rates using 3 different 4 th order diagonal-norm SBP operators. The first (columns 2-3) shows the traditional diagonal-norm, the second (columns 4-5) using 6 boundary stencils on equidistant grid, and the third (columns 6-7) minimizing with 5 boundary points and 3 non-equidistant grid locations.

\begin{tabular}{lllllll}
\hline$N$ & $\log l_{2}$ & $q$ & $\log l_{2}$ & $q$ & $\log l_{2}$ & $q$ \\
\hline 51 & -1.22 & 0.00 & -1.11 & 0.00 & -2.26 & 0.00 \\
101 & -2.52 & 4.31 & -2.71 & 5.32 & -4.04 & 5.91 \\
201 & -3.92 & 4.65 & -4.18 & 4.88 & -5.81 & 5.88 \\
401 & -5.35 & 4.75 & -5.68 & 4.98 & -7.53 & 5.71 \\
801 & -6.71 & 4.54 & -7.19 & 5.00 & -9.16 & 5.42 \\
1201 & -7.47 & 4.29 & -8.07 & 5.00 & -10.08 & 5.24 \\
\hline
\end{tabular}

Table 2: $\log \left(l_{2}-\right.$ errors $)$ and convergence rates using 3 different 6 th order diagonal-norm SBP operators. The first (columns 2-3) shows the traditional diagonal-norm, the second (columns 4-5) using 8 boundary stencils on equidistant grid, and the third (columns 6-7) minimizing with 7 boundary points and 3 non-equidistant grid locations.

\begin{tabular}{lllllll}
\hline$N$ & $\log l_{2}$ & $q$ & $\log l_{2}$ & $q$ & $\log l_{2}$ & $q$ \\
\hline 51 & -1.20 & 0.00 & -3.16 & 0.00 & -1.08 & 0.00 \\
101 & -2.68 & 4.92 & -5.41 & 7.47 & -2.73 & 5.49 \\
201 & -4.21 & 5.10 & -7.37 & 6.49 & -4.51 & 5.92 \\
401 & -5.76 & 5.13 & -9.04 & 5.55 & -6.31 & 5.97 \\
801 & -7.24 & 4.91 & -10.60 & 5.20 & -8.14 & 6.08 \\
1201 & -8.04 & 4.58 & -11.46 & 4.89 & -9.21 & 6.09 \\
\hline
\end{tabular}

Table 3: $\log \left(l_{2}-\right.$ errors $)$ and convergence rates using 2 different 8 th order diagonal-norm SBP operators. The first (columns 2-3) shows the traditional diagonal-norm, the second (columns 4-5) using 8 boundary stencils and 3 non-equidistant grid locations. The third (columns 6-7) shows the 10 th order diagonal-norm with 11 boundary points on equal stretching. 


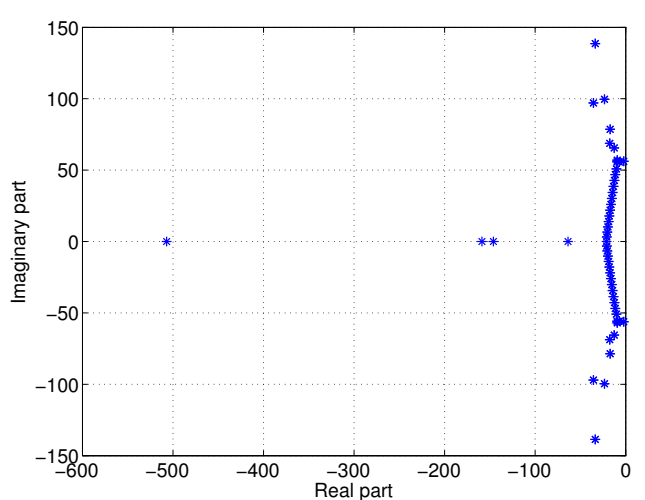

(a) Diagonal-norm SBP, $\lambda_{\max }=-2.21$

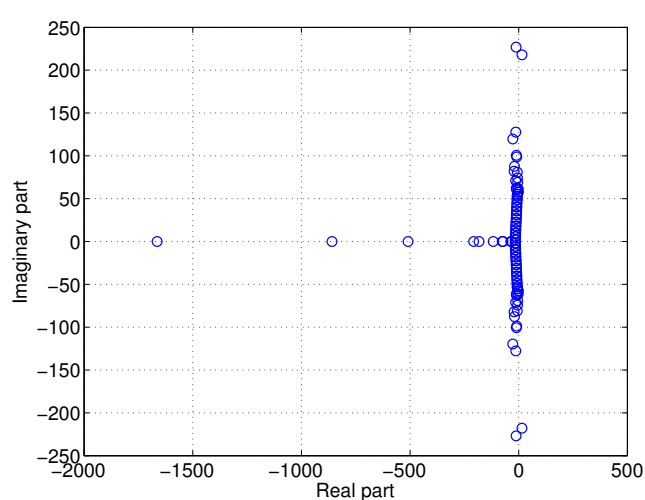

(b) Block-norm SBP, $\lambda_{\max }=15.02$

Figure 3: The spectrum to (6), where the system is defined by (10), comparing the 8 th order optimal diagonal-norm SBP operator and the corresponding 8th order block-norm SBP operator. $m=51 . \lambda_{\max }$ represents the largest real part of the spectrum.

\subsection{Second order systems}

We study the second order wave equation on piecewise discontinuous media,

$$
\begin{array}{lll}
a^{(l)} u_{t t}^{(l)}=\left(b^{(l)} u_{x}^{(l)}\right)_{x}, & -1 \leq x \leq 0, & t \geq 0 \\
a^{(r)} u_{t t}^{(r)}=\left(b^{(r)} u_{x}^{(r)}\right)_{x}, & 0 \leq x \leq 1, & t \geq 0 \\
b^{(l)} u_{x}^{(l)}=b^{(r)} u_{x}^{(r)}, u^{(l)}=u^{(r)}, & x=0, & t \geq 0 \\
u_{x}^{(l)}=0, & x=-1, & t \geq 0 \\
u_{x}^{(r)}=0, & x=1, & t \geq 0 \\
u^{(l)}=f^{(l)}, u_{t}^{(l)}=0, & -1 \leq x \leq 0, & t=0 \\
u^{(r)}=f^{(r)}, u_{t}^{(r)}=0, & 0 \leq x \leq 1, & t=0
\end{array},
$$

where $a^{(l)} \neq a^{(r)}, b^{(l)} \neq b^{(r)}$. Here $u^{(l, r)}$ denote the solutions corresponding to the left and right domains respectively, and $f^{(l, r)}$ the corresponding initial data. (A detailed analysis of this particular problem is described in [27].) We introduce the following coordinate transformations,

$$
\begin{array}{ll}
x=x(\xi)=\xi\left(\frac{e^{-\left(\xi+\frac{4}{5}\right)^{2}}}{e^{-\left(-1+\frac{4}{5}\right)^{2}}}\right)^{l}, & \xi \in[-1,0], \\
x=x(\xi)=\xi\left(\frac{e^{-\left(\xi-\frac{4}{5}\right)^{2}}}{e^{-\left(1-\frac{4}{5}\right)^{2}}}\right)^{l}, & \xi \in[0,1],
\end{array}
$$

in the left and right domains, respectively. Here $l$ is a non-negative integer, where the case $l=0$ corresponds to the Cartesian case. A larger value of $l$ 
leads to a denser clustering of grid-points close to the interface and the outer boundaries. The problem (13) transforms to

$$
\begin{array}{lll}
\tilde{a}^{(l)} u_{t t}^{(l)}=\left(\tilde{b}^{(l)} u_{\xi}^{(l)}\right)_{\xi}, & -1 \leq \xi \leq 0, & t \geq 0 \\
\tilde{a}^{(r)} u_{t t}^{(r)}=\left(\tilde{b}^{(r)} u_{\xi}^{(r)}\right)_{\xi}, & 0 \leq \xi \leq 1, & t \geq 0 \\
\tilde{b}^{(l)} u_{\xi}^{(l)}=\tilde{b}^{(r)} u_{\xi}^{(r)}, u^{(l)}=u^{(r)}, & \xi=0, & t \geq 0 \\
u_{\xi}^{(l)}=0, & \xi=-1, & t \geq 0 \\
u_{\xi}^{(r)}=0, & \xi=1, & t \geq 0 \\
u^{(l)}=f^{(l)}, u_{t}^{(l)}=0, & -1 \leq \xi \leq 0, & t=0 \\
u^{(r)}=f^{(r)}, u_{t}^{(r)}=0, & 0 \leq \xi \leq 1, & t=0
\end{array}
$$

in curvilinear coordinates, where $\tilde{a}^{(l, r)}=a^{(l, r)} x_{\xi}^{(l, r)}$ and $\tilde{b}^{(l, r)}=b^{(l, r)} / x_{\xi}^{(l, r)}$.

The semi-discrete approximation of the continuity conditions $u^{(l)}=u^{(r)}$ and $\tilde{b}^{(l)} u_{\xi}^{(l)}=\tilde{b}^{(r)} u_{\xi}^{(r)}$ can be written,

$$
I_{1} \equiv e_{m}^{T} v^{(l)}-e_{1}^{T} v^{(r)}=0, \quad I_{2} \equiv \tilde{B}^{(l)} e_{m} D_{1} v^{(l)}-\tilde{B}^{(r)} e_{1} D_{1} v^{(r)}=0
$$

where $v^{(l, r)}$ are the solution vectors corresponding to the left and right domains respectively, and $\tilde{B}^{(l, r)}$ are the projections of $\tilde{b}^{l, r}(\xi)$ onto the diagonals. The left and right domains are discretized using $(m)$ grid points.

The SBP-SAT method for (15) is given by,

$$
\begin{array}{rlrl}
\tilde{A}^{(l)} v_{t t}^{(l)} & =D_{1} \tilde{B}^{(l)} D_{1} v^{(l)} & \tilde{A}^{(r)} v_{t t}^{(2)} & =D_{1} \tilde{B}^{(r)} D_{1} v^{(r)} \\
& +\tau H^{-1} e_{m}\left(I_{1}\right) & & -\tau H^{-1} e_{1}\left(I_{1}\right) \\
& +\beta H^{-1} \tilde{B}^{(l)}\left(e_{m} D_{1}\right)^{T}\left(I_{1}\right) & & -\beta H^{-1} \tilde{B}^{(l)}\left(e_{1} D_{1}\right)^{T}\left(I_{1}\right) \\
& +\gamma H^{-1} e_{m}\left(I_{2}\right) & -\gamma H^{-1} e_{1}\left(I_{2}\right) \\
& -H^{-1} \tilde{B}^{(l)} e_{1} D_{1} v^{(l)} & & +H^{-1} \tilde{B}^{(r)} e_{m} D_{1} v^{(r)}
\end{array}
$$

The following Lemma was first stated in [27]:

Lemma 3.1 The scheme (17) is strictly stable if $D_{1}$ is a diagonal-norm SBP operators, $\gamma=-\frac{1}{2}, \beta=\frac{1}{2}$ and $\tau \leq-\frac{\tilde{b}^{(l)}+\tilde{b}^{(r)}}{4 / 5 h}$ hold.

The proof can be found in [27].

To show the importance of using diagonal-norm SBP operators for this problem we present in Figure 4 the eigenvalues to (17), on a grid defined by (14) with $l=2$ and $m=51$. We compare the spectra using the optimal 8th order diagonal-norm SBP operator and the corresponding block-norm 


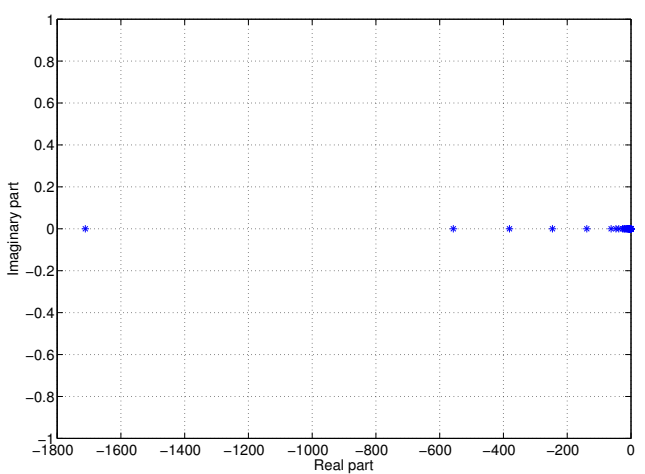

(a) Diagonal-norm SBP

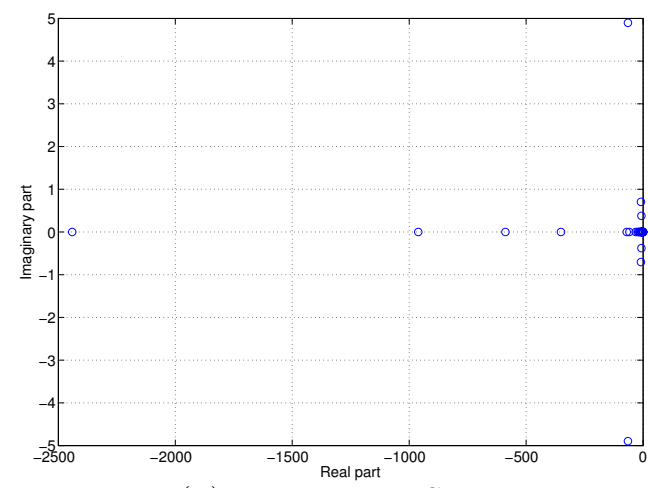

(b) Block-norm SBP

Figure 4: The spectrum to (17) on a grid defined by (14) with $l=2$ and $m=51$. Comparing the optimal diagonal $8 t h$ order SBP operator and the corresponding 8 th order block-norm SBP operator. Imaginary eigenvalues lead to instability.

SBP operator. Stability can not be guaranteed on curvilinear grids using block-norm SBP operators. (Stability requires that all eigenvalues are real and non-positive.)

The accuracy properties will be tested for an analytic standing wave solution, across a discontinuous media interface (located at $\xi=0$ ),

$$
\begin{array}{ll}
u^{(l)}=\cos \left(\pi \sqrt{b^{(l)} a^{(l)}} t\right) \cos \left(\pi a^{(l)} \xi\right), & \xi \in[-1,0], \\
u^{(r)}=\cos \left(\pi \sqrt{b^{(r)} a^{(r)}} t\right) \cos \left(\pi a^{(r)} \xi\right), & \xi \in[0,1] .
\end{array}
$$

The analytic solution is constructed such that $u_{\xi} \neq 0$ at the interface $(\xi=0)$ by choosing $a^{(l)}=15+\frac{1}{2}, a^{(r)}=5+\frac{1}{2}, b^{(l)}=a^{(r)}$ and $b^{(r)}=a^{(l)}$. At the outer boundaries we impose homogeneous Neumann boundary conditions. The numerical approximations are integrated to $t=1$ using the $4 t h$ order RungeKutta method, with a time step $d t=0.06 h$. (There is little difference between the time-step restrictions using the traditional and optimal SBP operators.)

In Tables 4 and 5 we compare the result using the traditional and optimal diagonal-norm SBP operators. We compare the convergence on both an equidistant grid, by setting $l=0$ in (14), and a stretched grid, by setting $l=2$ in (14). In Figure 5 we present the piecewise error using the two different 8 th order SBP operators, corresponding to the results using $m=51$ in Table 5. 


\begin{tabular}{lllllllll}
\hline$N$ & $\log l_{2}^{(l=0)}$ & $q$ & $\log l_{2}^{(l=0)}$ & $q$ & $\log l_{2}^{(l=2)}$ & $q$ & $\log l_{2}^{(l=2)}$ & $q$ \\
\hline 51 & -0.56 & 0.00 & -1.44 & 0.00 & -0.69 & 0.00 & -0.64 & 0.00 \\
101 & -1.84 & 4.29 & -3.21 & 5.98 & -2.44 & 5.90 & -2.42 & 5.99 \\
201 & -3.17 & 4.47 & -4.99 & 5.94 & -4.21 & 5.93 & -4.21 & 6.00 \\
401 & -4.64 & 4.91 & -6.71 & 5.75 & -5.94 & 5.78 & -6.02 & 6.02 \\
801 & -6.03 & 4.60 & -8.33 & 5.38 & -7.45 & 5.00 & -7.82 & 6.02 \\
\hline
\end{tabular}

Table 4: $\log \left(l_{2}-\right.$ errors $)$ and convergence rates. Comparing the traditional (columns 2-3) and optimal (columns 4-5) 6th order diagonal-norm SBP operators on non-stretched grids, $l=0$ in (14). The last four columns show the traditional and optimal $6 t h$ order diagonal-norm on a stretched grid, $l=2$ in (14).

\begin{tabular}{lllllllll}
\hline$N$ & $\log l_{2}^{(l=0)}$ & $q$ & $\log l_{2}^{(l=0)}$ & $q$ & $\log l_{2}^{(l=2)}$ & $q$ & $\log l_{2}^{(l=2)}$ & $q$ \\
\hline 51 & -0.57 & 0.00 & -2.10 & 0.00 & -1.09 & 0.00 & -1.06 & 0.00 \\
101 & -2.16 & 5.39 & -4.30 & 7.41 & -2.99 & 6.40 & -3.39 & 7.87 \\
201 & -3.33 & 3.91 & -6.34 & 6.85 & -4.74 & 5.83 & -5.79 & 8.02 \\
401 & -5.08 & 5.84 & -8.11 & 5.88 & -5.97 & 4.11 & -8.21 & 8.05 \\
801 & -6.37 & 4.27 & -9.71 & 5.34 & -7.72 & 5.83 & -10.62 & 8.04 \\
\hline
\end{tabular}

Table 5: $\log \left(l_{2}-\right.$ errors $)$ and convergence rates. Comparing the traditional (columns 2-3) and optimal (columns 4-5) 8th order diagonal-norm SBP operators on non-stretched grids, $l=0$ in (14). The last four columns show the traditional and optimal 8th order diagonal-norm on a stretched grid, $l=2$ in (14). 


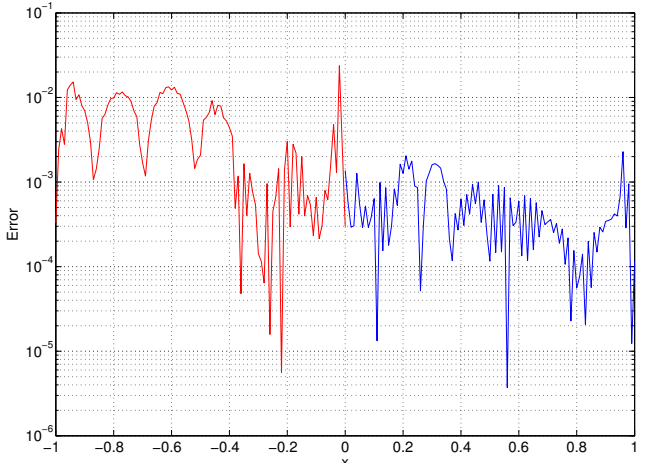

(a) Traditional 8 th order SBP

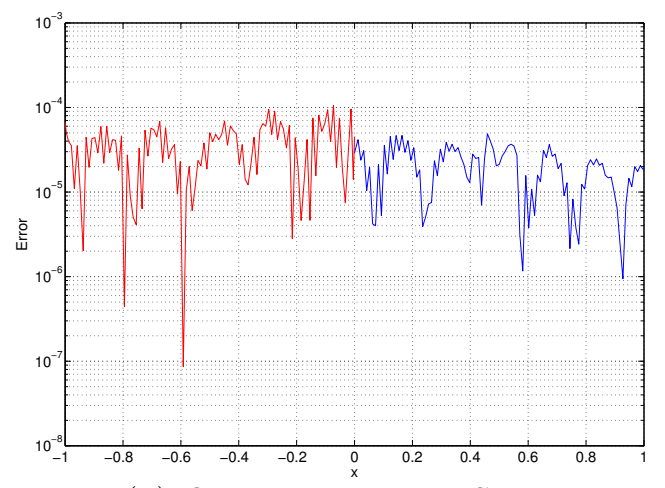

(b) Optimal 8 th order SBP

Figure 5: The point-wise error at $t=1$, solving (17) on a grid defined by (14) with $l=0$ and $m=51$, comparing the traditional and optimal 8th order diagonal-norm SBP operator. Notice the different scales.

\section{The Euler vortex problem}

To test the accuracy properties of the new optimal diagonal-norm SBP operators in a more challenging 2-D application, we consider the nonlinear Euler equations (see for example [37]) in curvilinear coordinates formulated in conservative form

$$
J \mathbf{u}_{t}+\tilde{\mathbf{F}}_{\xi}+\tilde{\mathbf{G}}_{\eta}=0,
$$

where

$$
\mathbf{u}=\left[\begin{array}{c}
\rho \\
\rho u \\
\rho v \\
e
\end{array}\right], \tilde{\mathbf{F}}=\left[\begin{array}{c}
\rho U \\
\rho u U+y_{\eta} p \\
\rho v U-x_{\eta} p \\
(e+p) U
\end{array}\right], \tilde{\mathbf{G}}=\left[\begin{array}{c}
\rho V \\
\rho u V-y_{\xi} p \\
\rho v V+x_{\xi} p \\
(e+p) V
\end{array}\right]
$$

Here

$$
\begin{gathered}
J=x_{\xi} y_{\eta}-x_{\eta} y_{\xi}, \\
U=y_{\eta} u-x_{\eta} v, V=-y_{\xi}+x_{\xi} v .
\end{gathered}
$$

$\rho$ is the density (scaled to free stream density $\rho_{\infty}$ ); $u, v$ are the velocity components in the $x, y$ directions respectively (scaled to free stream speed of sound $\left.c_{\infty}\right) ; e=e_{i}+\frac{\rho}{2}\left(u^{2}+v^{2}\right)$ the total energy (nondimensionalized with respect to $\rho_{\infty} c_{\infty}^{2}$ ) and $e_{i}$ is the internal energy. Pressure is obtained from the equation of state for a perfect gas $p=(\gamma-1) e_{i}$.

In the application below we consider the propagation of a vortex that satisfies the two-dimensional Euler equations, under the assumption of isentropy. The analytic vortex solution is steady in the frame of reference moving 
with the free-stream. The scaled vortex has the velocity field

$$
v_{\Theta}=\frac{\epsilon r}{2 \pi} \exp \left(\frac{1-r^{2}}{2}\right)
$$

where $\epsilon$ is the nondimensional circulation, $(r, \Theta)$ are the polar coordinates. The analytic solution in a fixed frame of reference $(x, y, t)$ becomes

$$
\begin{array}{ll}
u=1-\frac{\epsilon y}{2 \pi} \exp \left(\frac{f(x, y, t)}{2}\right), & v=\frac{\epsilon\left(\left(x-x_{0}\right)-t\right)}{2 \pi} \exp \left(\frac{f(x, y, t)}{2}\right) \\
\rho=\left(1-\frac{\epsilon^{2}(\gamma-1) M^{2}}{8 \pi^{2}} \exp (f(x, y, t))\right)^{\frac{1}{\gamma-1}}, & p=\frac{\rho^{\gamma}}{\gamma M^{2}}
\end{array}
$$

where $f(x, y, t)=1-\left(\left(\left(x-x_{0}\right)-t\right)^{2}+y^{2}\right) ; M$ is the Mach number; $\gamma=$ $c_{p} / c_{v}=1.4$ and $x_{0}$ is the initial position of the vortex (in the $x$-direction). The vortex is introduced into the computational domain by using the analytic solution as boundary data and initial data.

The main focus is to verify the accuracy for a non-smooth multi-block interface coupling. Hence, we will solve the Euler-vortex problems on a twoblock domain (see Figures 7 and 7). Details concerning how to discretize this problem with the SBP-SAT method can be found in [44, 31, 25].

\subsection{Computations}

In the first two tests we verify the convergence properties using non-stretched grids (see Figure 7) and stretched grids (see Figures 8 ). The vortex in initiated (at $t=0)$, centered at $(x, y)=(0,-0.5)$, i.e., at the grid-interface between the two blocks (where there is a discontinuity in the metrics). The vortex is then propagated (to the right) along the grid interface a distance of 4 units (corresponding to $t=4$ ) where we measure the $l_{2}$-errors. The vortex is integrated using a 6th order accurate Runge-Kutta method [2] using a CFL of 0.4. We set the Mach number $M=0.5$.

In Tables 6 and 7 we present the results using the traditional diagonalnorm and optimal diagonal-norm SBP operators where we apply no stretching, corresponding to $l=0$ in (14) in both the $\mathrm{x}$ - and y-direction, see Figure 7. We performed a detailed study (although not presented here) of what would be the optimal (in terms of accuracy) grid-stretching for the traditional 8th order diagonal SBP operator for this 2-block grid. This study yielded a stretching corresponding to $l=2$ in (14) in both the $\mathrm{x}$ - and $\mathrm{y}$ direction. In Tables 9 and 10 we present the results using the traditional diagonal-norm and optimal diagonal-norm SBP operators where we apply the optimal grid-stretching corresponding to $l=2$ in (14) in both the $\mathrm{x}$ - and 
y-direction, see Figure 7. In Table we list the CFL constraint (relative the second order accurate traditional SBP scheme on a non-stretched grid) for the traditional and optimal SBP schemes for the Euler vortex problem, for both the non-stretched grid, $l=0$ in (14) and the stretched grid, $l=2$ in (14).

In Figure 6 we plot the $l_{2}$-errors (taken from Tables 6 and 7 ) versus the number of grid-points (in each dimension) for the traditional and optimal $6 t h$ and $8 t h$ order accurate SBP operators in a $\log -\log$ plot, to more clearly see the huge gain in efficiency going to optimal SBP operators.

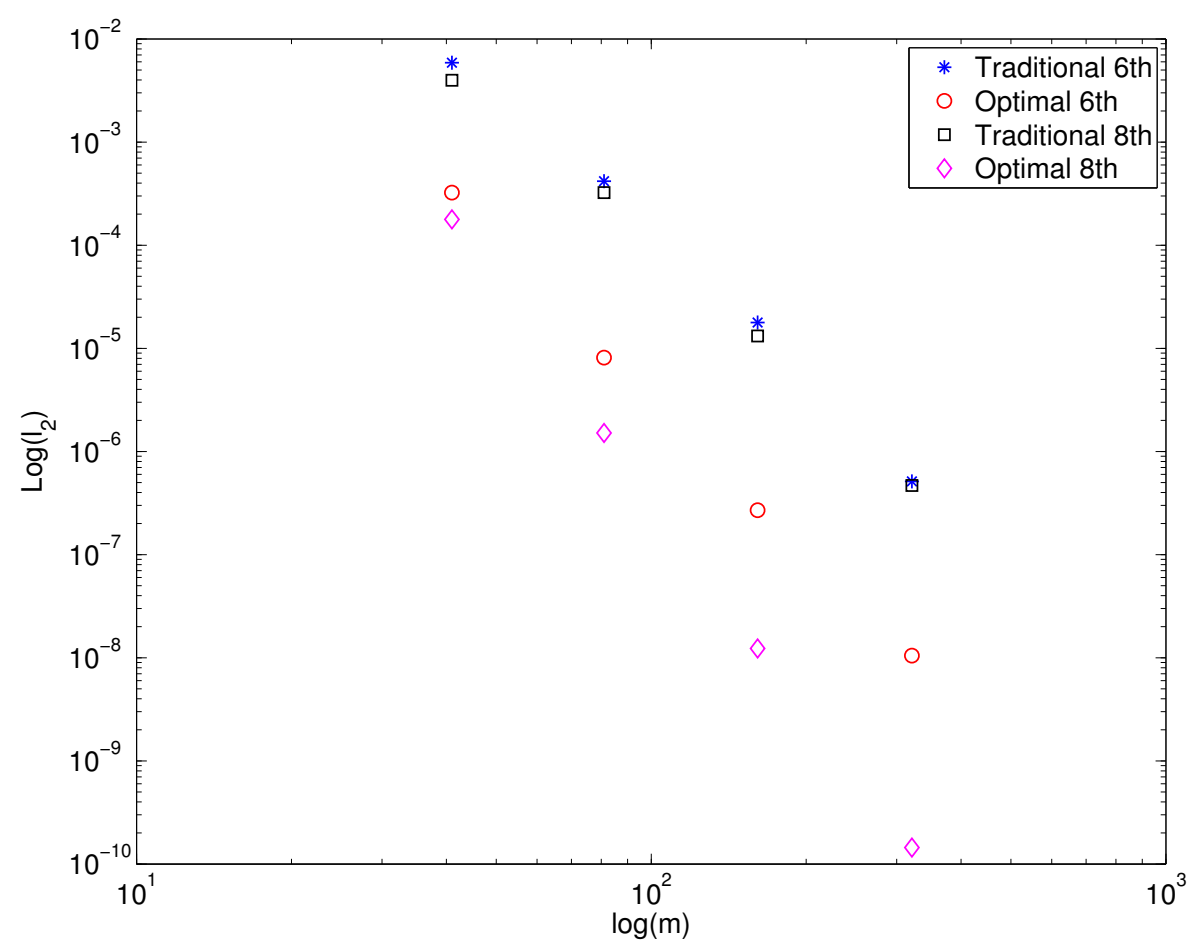

Figure 6: Comparing the traditional and optimal 6th and 8th order accurate SBP operators in a $\log -\log$ plot, results taken from Tables 6 and 7 .

Remark By comparing Tables 7 and 10 we see that we get good accuracy also on coarse grids using the optimal 6th and 8th order SBP operators and that there is no gain using a stretched grid for this particular problem. Furthermore, the stretching had some impact on the time-step restrictions (see Table 8) due to very small cells close to the boundaries. However, we do obtain a significant improvement in accuracy by stretching the grids to cluster the points towards the boundaries using the traditions SBP operators (see Tables 6 and 9). A fair comparison of efficiency is given by taking both the achieved accuracy and time-step restriction in consideration. 


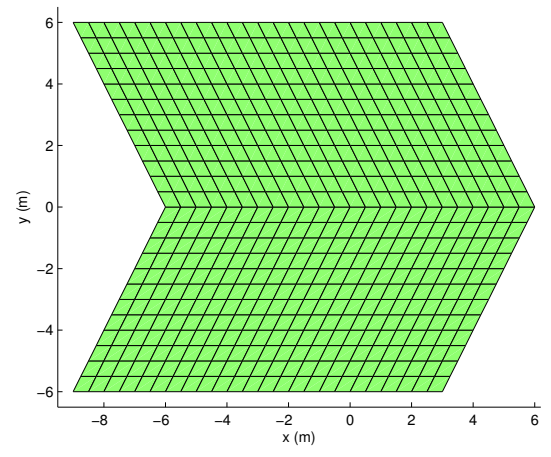

(a) Traditional SBP, $l=0$.

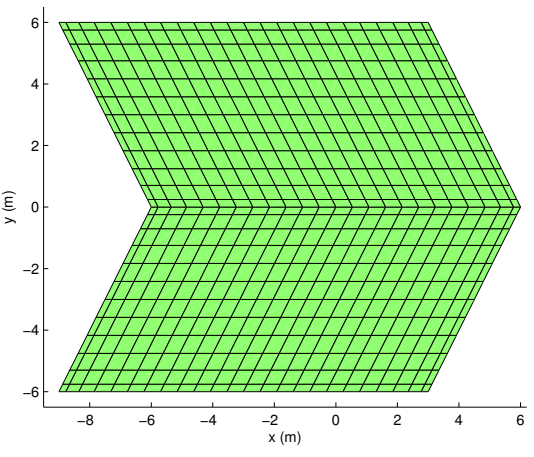

(b) Traditional SBP, $l=0$.

Figure 7: The computational domain, consisting of 2 curvilinear grids, with discontinuous metrics along the interface. Here with $21 \times 11$ grid-points in each domain and no stretching of the grids, $l=0$ in (14).

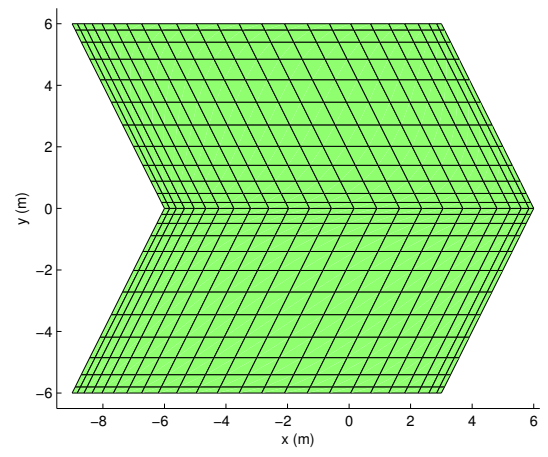

(a) Standard SBP, $l=2$.

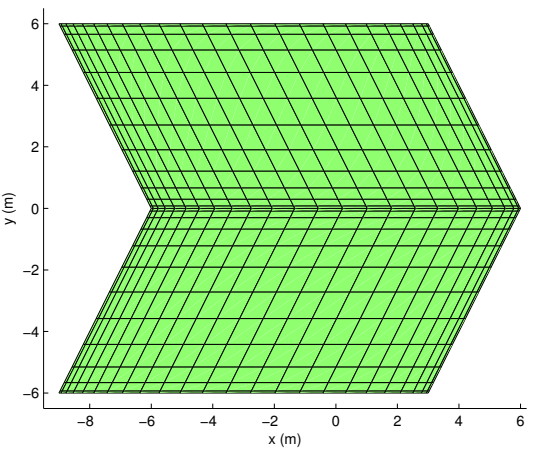

(b) Optimal SBP, $l=2$.

Figure 8: The computational domain, consisting of 2 curvilinear grids, with discontinuous metrics along the interface. Here with $21 \times 11$ grid-points in each domain and stretched grids, $l=2$ in (14). 


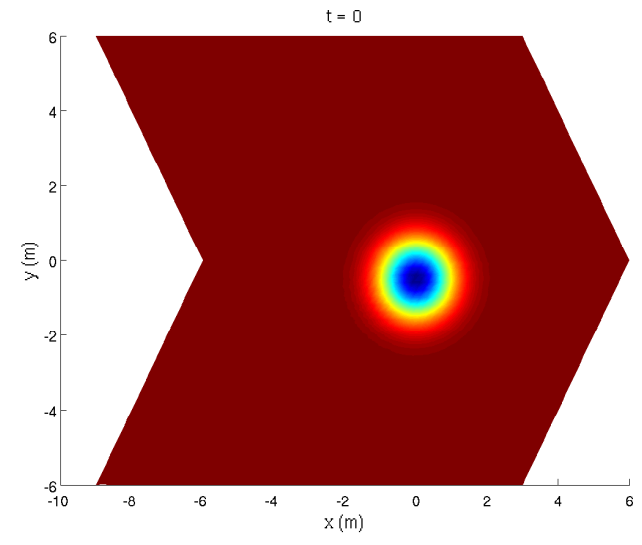

(a) Initial condition at $t=0$.

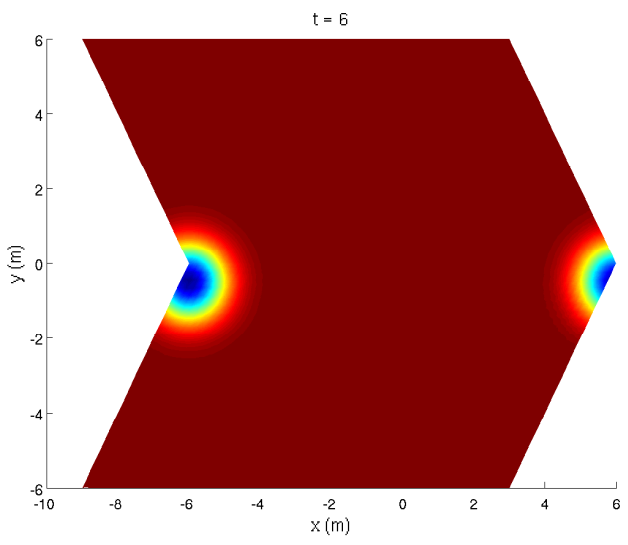

(b) Solution at $t=6$.

Figure 9: The solution (pressure) at $t=0$, and later at $t=6$ when the vortex reaches the outermost right boundary, and enters through the left boundary.

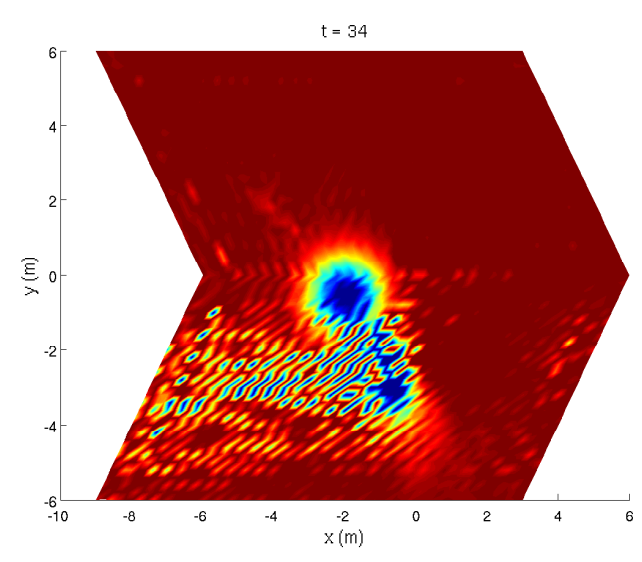

(a) Traditional SBP.

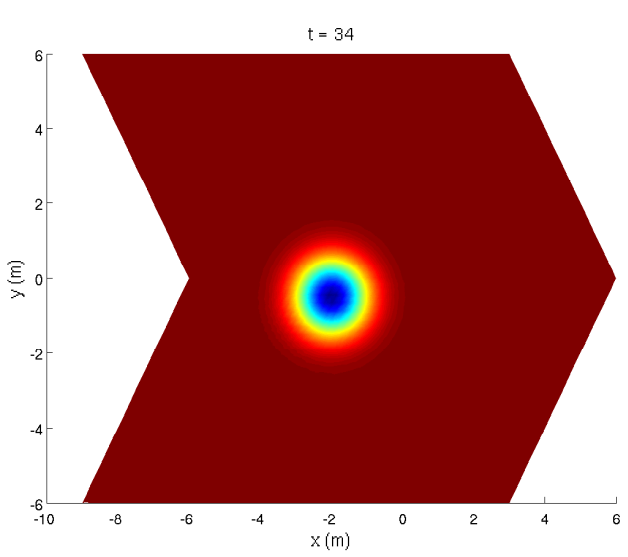

(b) Optimal SBP

Figure 10: The solution (pressure) at $t=34$ using $m=31 \times 61$ grid-points (in each sub-block), comparing the solutions with the traditional and optimal 8th order SBP.

To test the stability properties and also the superior accuracy of the optimal diagonal-norm SBP operators (compared to the traditional diagonalnorm SBP operators) we perform long-time simulations to $t=34$. The west and east boundaries are coupled using SAT, and it takes 12 units in time for the vortex to cross the domain (from left to right). At $t=34$ the vortex has crossed the west-east interface 3 times.

In Figure 10 we present the solutions for two different schemes: traditional 8th order diagonal-norm SBP and optimal 8th order diagonal-norm SBP, 


\begin{tabular}{cllllllll}
\hline$N_{x} \times N_{y}$ & $\log l_{2}{ }^{(2 n d)}$ & $q^{(2 n d)}$ & $\log l_{2}{ }^{(4 t h)}$ & $q^{(4 t h)}$ & $\log l_{2}{ }^{(6 t h)}$ & $q^{(6 t h)}$ & $\log l_{2}{ }^{(8 t h)}$ & $q^{(8 t h)}$ \\
\hline $41 \times 21$ & -2.41 & 0.00 & -2.70 & 0.00 & -2.23 & 0.00 & -2.40 & 0.00 \\
$81 \times 41$ & -3.06 & 2.17 & -3.69 & 3.29 & -3.38 & 3.81 & -3.49 & 3.60 \\
$161 \times 81$ & -3.66 & 2.00 & -4.67 & 3.25 & -4.75 & 4.58 & -4.88 & 4.62 \\
$321 \times 161$ & -4.27 & 2.00 & -5.60 & 3.09 & -6.29 & 5.09 & -6.33 & 4.82 \\
\hline
\end{tabular}

Table 6: $\log \left(l_{2}\right.$-errors $)$ and convergence rates using the traditional diagonalnorm operators with no grid stretching $l=0 . N_{x}$ and $N_{y}$ denote the number of grid points in the $x$ and $y$ directions, respectively, in each subdomain.

\begin{tabular}{cllllllll}
\hline$N_{x} \times N_{y}$ & $\log l_{2}{ }^{(2 n d)}$ & $q^{(2 n d)}$ & $\log l_{2}{ }^{(4 t h)}$ & $q^{(4 t h)}$ & $\log l_{2}^{(6 t h)}$ & $q^{(6 t h)}$ & $\log l_{2}^{(8 t h)}$ & $q^{(8 t h)}$ \\
\hline $41 \times 21$ & -2.51 & 0.00 & -3.45 & 0.00 & -3.49 & 0.00 & -3.75 & 0.00 \\
$81 \times 41$ & -3.12 & 2.02 & -4.63 & 3.91 & -5.09 & 5.33 & -5.82 & 6.89 \\
$161 \times 81$ & -3.71 & 1.98 & -5.72 & 3.63 & -6.57 & 4.91 & -7.91 & 6.93 \\
$321 \times 161$ & -4.31 & 1.99 & -6.84 & 3.71 & -7.98 & 4.66 & -9.84 & 6.43 \\
\hline
\end{tabular}

Table 7: $\log \left(l_{2}-\right.$ errors $)$ and convergence rates using the optimal diagonalnorm operators with no grid stretching $l=0 . N_{x}$ and $N_{y}$ denote the number of grid points in the $x$ and $y$ directions, respectively, in each subdomain.

using $31 \times 61$ grid-points in each subdomain. The optimal 8 th order diagonalnorm SBP operator yields much more accurate results. Both SBP operators are closed with $4 t h$ order accurate boundary stencils, and it is evident that the accuracy of the boundary stencils dictates the accuracy properties when block-interfaces (or boundaries) are present.

\section{Conclusions and future work}

The main focus has been to construct new diagonal-norm SBP operators with optimal accuracy properties, to combine truly high-order accuracy and stability on curvilinear multi-block grids. This is achieved by introducing more boundary stencils and (or) allowing the grid-points to be non-equidistant

\begin{tabular}{cllllllll}
\hline Operator & $2 n d^{l=0}$ & $4 t h^{l=0}$ & $6 t h^{l=0}$ & $8 t h^{l=0}$ & $2 n d^{l=2}$ & $4 t h^{l=2}$ & $6 t h^{l=2}$ & $8 t h^{l=2}$ \\
\hline traditional & 1 & 0.60 & 0.55 & 0.49 & 0.45 & 0.27 & 0.22 & 0.21 \\
optimal & 0.93 & 0.58 & 0.49 & 0.37 & 0.28 & 0.14 & 0.12 & 0.09 \\
\hline
\end{tabular}

Table 8: Maximal time step with no grid stretching $(l=0)$ and with $l=2$ in (14), relative the time-step for the $2 n d$ order scheme on a non-stretched grid. Determined experimentally on an $81 \times 41$ grid. 


\begin{tabular}{cllllllll}
\hline$N_{x} \times N_{y}$ & $\log l_{2}{ }^{(2 n d)}$ & $q^{(2 n d)}$ & $\log l_{2}{ }^{(4 t h)}$ & $q^{(4 t h)}$ & $\log l_{2}{ }^{(6 t h)}$ & $q^{(6 t h)}$ & $\log l_{2}{ }^{(8 t h)}$ & $q^{(8 t h)}$ \\
\hline $41 \times 21$ & -2.24 & 0.00 & -2.91 & 0.00 & -2.84 & 0.00 & -2.57 & 0.00 \\
$81 \times 41$ & -2.79 & 1.80 & -4.00 & 3.60 & -4.47 & 5.42 & -4.17 & 5.29 \\
$161 \times 81$ & -3.38 & 1.98 & -5.14 & 3.81 & -6.15 & 5.58 & -5.93 & 5.85 \\
$321 \times 161$ & -3.98 & 2.00 & -6.27 & 3.76 & -7.73 & 5.22 & -7.33 & 4.65 \\
\hline
\end{tabular}

Table 9: $\log \left(l_{2}\right.$-errors $)$ and convergence rates using the traditional diagonalnorm operators with grid stretching $l=2 . N_{x}$ and $N_{y}$ denote the number of grid points in the $x$ and $y$ directions, respectively, in each subdomain.

\begin{tabular}{cllllllll}
\hline$N_{x} \times N_{y}$ & $\log l_{2}{ }^{(2 n d)}$ & $q^{(2 n d)}$ & $\log l_{2}{ }^{(4 t h)}$ & $q^{(4 t h)}$ & $\log l_{2}^{(6 t h)}$ & $q^{(6 t h)}$ & $\log l_{2}^{(8 t h)}$ & $q^{(8 t h)}$ \\
\hline $41 \times 21$ & -2.24 & 0.00 & -2.93 & 0.00 & -3.27 & 0.00 & -3.46 & 0.00 \\
$81 \times 41$ & -2.78 & 1.81 & -4.02 & 3.59 & -4.88 & 5.34 & -5.62 & 7.17 \\
$161 \times 81$ & -3.38 & 1.99 & -5.18 & 3.87 & -6.64 & 5.85 & -7.97 & 7.80 \\
$321 \times 161$ & -3.98 & 2.00 & -6.38 & 3.97 & -8.43 & 5.96 & -10.36 & 7.97 \\
\hline
\end{tabular}

Table 10: $\log \left(l_{2}\right.$-errors $)$ and convergence rates using the optimal diagonalnorm operators with grid stretching $l=2 . N_{x}$ and $N_{y}$ denote the number of grid points in the $x$ and $y$ directions, respectively, in each subdomain.

close to the boundaries. The boundary and interface conditions are treated with the SAT technique. We study both first and second order hyperbolic problems on piecewise curvilinear multi-block grids.

Numerical computations in 1-D and 2-D corroborate the stability- and accuracy-properties and also show that the numerical schemes built on optimal diagonal-norm SBP operators are superior to the corresponding traditional diagonal-norm SBP operators. For the second order wave equation we obtain super-convergence, and also for the 2-D Euler vortex problem the new optimal SBP operators yield higher than expected order of convergence.

It is likely that further improvements in accuracy could have been achieved for the optimal 6th and 8th order cases by allowing for more than 3 "free" locations of the boundary points in the minimisation. However, due to a limitation in Maple we where forced to limit the number to 3. With other minimisation routines this limitation might be lifted. The 8th order case could potentially allow for 7 free boundary points.

In a coming study we will investigate the efficiency of using the new diagonal-norm SBP operators in a more complex 3-D setting, with focus on second order hyperbolic systems and hyperbolic-parabolic systems. 


\section{APPENDIX}

The boundary closures (the coefficients) are present for the $Q$ and $H$ matrices. Notice that the coefficients in the norm should be multiplied with the grid-step $h$ to have the correct normalization.

\section{SBP operators with additional boundary stencils on equidistant grids.}

\section{I.1 2nd order, 3 boundary stencils}

the upper part of the $\mathrm{Q}$

$$
Q_{1,2}=\frac{7}{12} \quad Q_{1,3}=-\frac{1}{12} \quad Q_{2,3}=-\frac{7}{12}
$$

The upper part of the norm

$$
H_{1,1}=\frac{5}{12} \quad H_{2,2}=\frac{7}{6} \quad H_{3,3}=\frac{11}{12}
$$

\section{I.2 Fourth order, 6 boundary stencils}

The upper part of the norm

$$
\begin{array}{lll}
H_{1,1}=\frac{511}{1600} & H_{3,3}=\frac{929}{1440} & H_{5,5}=\frac{2659}{2880} \\
H_{2,2}=\frac{3971}{2880} & H_{4,4}=\frac{587}{480} & H_{6,6}=\frac{14551}{14400}
\end{array}
$$

The upper part of $\mathrm{Q}$,

$$
\begin{array}{lll}
Q_{1,2}=\frac{436061}{680400} & Q_{2,3}=\frac{238831}{544320} & Q_{3,5}=-\frac{74827}{1088640} \\
Q_{1,3}=-\frac{312391}{5443200} & Q_{2,4}=\frac{290357}{1088640} & Q_{3,6}=\frac{3859}{907200} \\
Q_{1,4}=-\frac{100109}{907200} & Q_{2,5}=-\frac{125}{5184} & Q_{4,5}=\frac{342799}{544320} \\
Q_{1,5}=\frac{54107}{5443200} & Q_{2,6}=-\frac{220357}{5443200} & Q_{4,6}=-\frac{149959}{5443200} \\
Q_{1,6}=\frac{263}{15552} & Q_{3,4}=\frac{3467}{7776} & Q_{5,6}=\frac{428789}{680400}
\end{array}
$$

\section{I.3 Sixth order, 8 boundary stencils}

The upper part of the norm

$$
\begin{array}{lll}
H_{1,1}=\frac{7497391}{25401600} & H_{3,3}=\frac{105317}{403200} & H_{6,6}=\frac{513973}{403200} \\
H_{2,2}=\frac{1106227}{725760} & H_{4,4}=\frac{260179}{145152} & H_{7,7}=\frac{671171}{725760} \\
H_{5,5}=\frac{303631}{725760} & H_{8,8}=\frac{25631999}{25401600}
\end{array}
$$


Left boundary closure of $\mathrm{Q}$ ( 8 by 8 block) is given below:

$$
\begin{array}{lll}
Q_{1,2}=\frac{14085349}{21168000} & Q_{2,5}=\frac{1214239}{16934400} & Q_{4,5}=\frac{8120941}{30481920} \\
Q_{1,3}=-\frac{2505189}{8064000} & Q_{2,6}=-\frac{246914711}{762048000} & Q_{4,6}=\frac{3467971}{6350400} \\
Q_{1,4}=-\frac{7583609}{38102400} & Q_{2,7}=\frac{23380423}{304819200} & Q_{4,7}=-\frac{42123859}{304819200} \\
Q_{1,5}=-\frac{89573}{4233600} & Q_{2,8}=\frac{3}{200} & Q_{4,8}=-\frac{2075677}{152409600} \\
Q_{1,6}=\frac{9}{80} & Q_{3,4}=\frac{22302157}{101606400} & Q_{5,7}=\frac{1526309607}{304819200} \\
Q_{1,7}=-\frac{39279943}{1524096000} & Q_{3,5}=-\frac{6048877}{60963840} & Q_{5,8}=-\frac{81127}{3386880} \\
Q_{1,8}=-\frac{13}{20000} & Q_{3,6}=\frac{9737351}{169344000} & Q_{6,7}=\frac{321139631}{508032000} \\
Q_{2,3}=\frac{56626691}{304819200} & Q_{3,7}=-\frac{500309}{25401600} & Q_{6,8}=-\frac{49594423}{762048000} \\
Q_{2,4}=\frac{97579603}{152409600} & Q_{3,8}=\frac{780937}{304819200} & Q_{7,8}=\frac{30841499}{43545600}
\end{array}
$$

\section{SBP operators, with three non-equidistant grid-points.}

Let $\mathbf{x} \in[0,1]$. Introduce $d=d_{1}+d_{2}+d_{3}$, where $h d_{1}$ is the distance between the first and second grid-point, $h d_{2}$ is the distance between the second and third grid-point, and $h d_{3}$ is the distance between the third and fourth gridpoint. Here $h=1 /(2 d+(m-7))$ and $m$ is the number of grid-points. The grid-points are located at

$$
\begin{gathered}
\mathbf{x}=\left[0, d_{1} h,\left(d_{1}+d_{2}\right) h, d h,(d+1) h,(d+2) h, \ldots,\right. \\
\left.(d+(m-7)) h, 1-d h, 1-\left(d_{1}+d_{2}\right) h, 1-d_{1} h, 1\right] .
\end{gathered}
$$

\section{II.1 2nd order, 3 boundary stencils and 2 non-equidistant grid-points.}

The first two grid-stretchings

$d_{1}=0.78866488858096586513, d_{2}=0.95915098594220826013, d_{3}=1$.

The upper part of the norm

$$
\begin{aligned}
& H_{1,1}=0.33743097329453577701 \\
& H_{2,2}=0.97759682018833491296
\end{aligned} \quad H_{3,3}=0.93278808104030343530
$$


The upper part of the $\mathrm{Q}$

$$
\begin{aligned}
& Q_{1,2}=0.55932483188770411252 \\
& Q_{1,3}=-0.05932483188770411252 \quad Q_{2,3}=0.55932483188770411252
\end{aligned}
$$

\section{2 4th order, 5 boundary stencils and 3 non-equidistant grid-points.}

The first three grid-stretchings

$$
\begin{aligned}
& d_{1}=0.72181367003646814327, \\
& d_{2}=1.3409118421582217252
\end{aligned} \quad d_{3}=1.2898797485951900258
$$

The upper part of the norm

$$
\begin{array}{ll}
H_{1,1}=0.21427296612044126417 & H_{3,3}=1.434458792494126 \\
H_{2,2}=1.123759588488739348 & H_{4,4}=1.0917323021736130836 \\
H_{5,5}=0.9883816115129601975
\end{array}
$$

The upper part of the $\mathrm{Q}$

$$
\begin{array}{ll}
Q_{1,2}=0.66884898686930380508 & Q_{2,4}=-0.32412368653542520402 \\
Q_{1,3}=-0.25171531878753856238 & Q_{2,5}=0.070828303918324098284 \\
Q_{1,4}=0.10997619816825822803 & Q_{3,4}=0.8180378089216779335 \\
Q_{1,5}=-0.027109866250023470592 & Q_{3,5}=-0.14760875822281158529 \\
Q_{2,3}=0.92214436948640491071 & Q_{4,5}=0.68722365388784429092
\end{array}
$$

\section{II.3 6th order, 7 boundary stencils and 3 non-equidistant grid-points.}

The first three grid-stretchings

$$
\begin{aligned}
& d_{1}=0.51670081689316731234, \\
& d_{2}=0.98190527037374634269
\end{aligned} \quad d_{3}=1.0868393364992957832
$$


The upper part of the norm

$$
\begin{aligned}
& H_{1,1}=0.15109714532036117328 \\
& H_{2,2}=0.80967585357107013003 \\
& H_{3,3}=1.0911427148079254850
\end{aligned}
$$

$$
\begin{aligned}
& H_{4,4}=1.0435269041571577756 \\
& H_{5,5}=0.98680905919946100728 \\
& H_{6,6}=1.0037581831426163456 \\
& H_{7,7}=0.99943556356761752125
\end{aligned}
$$

The upper part of the Q

$$
\begin{aligned}
& Q_{1,2}=0.66670790901888837033 \\
& Q_{1,3}=-0.23418791580399147484 \\
& Q_{1,4}=0.084251264588860596867 \\
& Q_{1,5}=-0.015923290838179674350 \\
& Q_{1,6}=-0.0015653772860347171721 \\
& Q_{1,7}=0.00071741032045689717567 \\
& Q_{2,3}=0.89405599296515541581 \\
& Q_{2,4}=-0.28597427787314667440 \\
& Q_{2,5}=0.057056178538117177397 \\
& Q_{2,6}=0.0041320613074890940489 \\
& Q_{2,7}=-0.0025620459187266476645
\end{aligned}
$$

$Q_{3,4}=0.82961715259707113283$ $Q_{3,5}=--0.18233747042994439227$ $Q_{3,6}=0.0083784382166533084621$ $Q_{3,7}=0.0042099567773838744673$ $Q_{4,5}=0.75419218459746682761$ $Q_{4,6}=-0.14034899831339963049$ $Q_{4,7}=0.014050953028717865444$ $Q_{5,6}=0.74751473989919011204$ $Q_{5,7}=-0.15119380469839682133$ $Q_{6,7}=0.75144419715723149872$

\section{II.4 8th order, 8 boundary stencils and 3 non-equidistant grid-points.}

The first three grid-stretchings

$$
\begin{aligned}
& d_{1}=0.41669687672575697416, \\
& d_{2}=0.78703773886730090312
\end{aligned} \quad d_{3}=0.92685925671601406028
$$

The upper part of the norm

$$
\begin{array}{ll}
H_{1,1}=0.12163222110707502878 & H_{5,5}=1.0072514376844677230 \\
H_{2,2}=0.65235832636546639982 & H_{6,6}=0.99768726657776478834 \\
H_{3,3}=0.87730414198101010954 & H_{7,7}=1.0005302998791085514 \\
H_{4,4}=0.97388951771079542799 & H_{8,8}=0.99994066100338390832
\end{array}
$$


The upper part of the $\mathrm{Q}$

$$
\begin{array}{ll}
Q_{1,2}=0.66670790901888837033 & Q_{3,5}=-0.21014872891771196683 \\
Q_{1,3}=-0.21994030190635039046 & Q_{3,6}=0.038572177503610408523 \\
Q_{1,4}=0.061752567584332553851 & Q_{3,7}=-0.0016637807199883547459 \\
Q_{1,5}=-0.0032312350944133128873 & Q_{3,8}=-0.00046321740653650899692 \\
Q_{1,6}=-0.0033934980320003350186 & Q_{4,5}=0.81102837324727866266 \\
Q_{1,7}=0.000015157027970563223705 & Q_{4,6}=-0.20423896795865859484 \\
Q_{1,8}=0.00032350133072942893419 & Q_{4,7}=0.034947121761434371005 \\
Q_{2,3}=0.86688767821045233147 & Q_{4,8}=0.0023139100244270367378 \\
Q_{2,4}=-0.24298087640343350527 & Q_{5,6}=0.80054065093594950025 \\
Q_{2,5}=0.039549469619698650847 & Q_{5,7}=-0.19699167992690472530 \\
Q_{2,6}=0.0020763528371484737510 & Q_{5,8}=0.037220336417235830241 \\
Q_{2,7}=-0.00065045489396961912976 & Q_{6,7}=0.79903079313046586260 \\
Q_{2,8}=-0.00040836028016484152445 & Q_{6,8}=-0.19999788736822592697 \\
& Q_{7,8}=0.80016334685519857774
\end{array}
$$$$
Q_{3,4}=0.82065092584472835146
$$

\section{References}

[1] S. Abarbanel and A. Ditkowski. Asymptotically stable fourth-order accurate schemes for the diffusion equation on complex shapes. J. Comput. Phys., 133:279-288, 1997.

[2] E. A. Alshinaa, E. M. Zaksb, and N. N. Kalitkin. Optimal first- to sixth-order accurate runge-kutta schemes. Computational Mathematics and Mathematical Physics, 48:395405, 2008.

[3] A. Bayliss, K. E. Jordan, B. J. Lemesurier, and E. Turkel. A fourth order accurate finite difference scheme for the computation of elastic waves. Bull. Seismol. Soc. Amer., 76(4):1115-1132, 1986.

[4] M. H. Carpenter, D. Gottlieb, and S. Abarbanel. The stability of numerical boundary treatments for compact high-order finite difference schemes. J. Comput. Phys., 108(2), 1993. 
[5] M. H. Carpenter, D. Gottlieb, and S. Abarbanel. Time-stable boundary conditions for finite-difference schemes solving hyperbolic systems: Methodology and application to high-order compact schemes. J. Comput. Phys., 111(2), 1994.

[6] M. H. Carpenter, J. Nordström, and D. Gottlieb. A Stable and Conservative Interface Treatment of Arbitrary Spatial Accuracy. J. Comput. Phys., 148, 1999.

[7] Mark H. Carpenter and Travis C. Fisher. High-Order Entropy Stable Formulations for Computational Fluid Dynamics. American Institute of Aeronautics and Astronautics, 2013/06/27 2013.

[8] Mark H. Carpenter and David Gottlieb. Spectral methods on arbitrary grids. Journal of Computational Physics, 129:74-86, 1996.

[9] Peter Diener, Ernst Nils Dorband, Erik Schnetter, and Manuel Tiglio. Optimized high-order derivative and dissipation operators satisfying summation by parts, and applications in three-dimensional multi-block evolutions. J. Sci. Comput., 32(1):109-145, 2007.

[10] Travis H. Fisher and Mark H. Carpenter. High-order entropy stable finite difference schemes for nonlinear conservation laws: Finite domains. J. Comput. Phys., 252:518-557, 2013.

[11] B. Gustafsson. The convergence rate for difference approximations to general mixed initial boundary value problems. SIAM J. Numer. Anal., 18(2):179-190, Apr. 1981.

[12] B. Gustafsson, H.-O. Kreiss, and J. Oliger. Time dependent problems and difference methods. John Wiley \& Sons, Inc., 1995.

[13] B. Gustafsson, H. O. Kreiss, and A. Sundström. Stability theory of difference approximations for mixed initial boundary value problems. Math. Comp., 26(119), 1972.

[14] B. Gustafsson and P. Olsson. Fourth-order difference methods for hyperbolic IBVPs. J. Comput. Physics, 117(1), 1995.

[15] J.S. Hesthaven. A stable penalty method for the compressible Navier-Stokes equations: II Multi-dimensional domain decomposition schmemes. SIAM J.Sci.Comput., 1998. 
[16] J. Hicken and D. Zingg. Superconvergent functional estimates from summation-by-parts finite-difference discretizations. SIAM Journal on Scientific Computing, 33(2):893-922, 2011.

[17] J. E. Hicken and D. W. Zingg. Parallel newton-krylov solver for the euler equations discretized using simultaneous approximation terms. AIAA Journal, 46(11):2773-2786, 2013/02/09 2008.

[18] Jason E. Hicken and David W. Zingg. Aerodynamic optimization algorithm with integrated geometry parameterization and mesh movement. AIAA Journal, 48(2):400-413, 2013/02/09 2010.

[19] J.E. Hicken. Output error estimation for summation-by-parts finitedifference schemes. Journal of Computational Physics, 231(9):3828 $3848,2012$.

[20] H.-O. Kreiss and G. Scherer. Finite element and finite difference methods for hyperbolic partial differential equations. Mathematical Aspects of Finite Elements in Partial Differential Equations., Academic Press, Inc., 1974.

[21] H.-O. Kreiss and G. Scherer. On the existence of energy estimates for difference approximations for hyperbolic systems. Technical report, Dept. of Scientific Computing, Uppsala University, 1977.

[22] Heinz-Otto Kreiss and Joseph Oliger. Comparison of accurate methods for the integration of hyperbolic equations. Tellus XXIV, 3, 1972.

[23] L. Lehner, O. Reula, and M. Tiglio. Multi-block simulations in general relativity: high-order discretizations, numerical stability and applications. Classical Quantum Gravity, 22:5283-5321, 2005.

[24] S. K. Lele. Compact finite difference schemes with spectral-like resolution. J. Comput. Phys., 103:16-42, 1992.

[25] K. Mattsson and M. Almqvist. A solution to the stability issues with block norm summation by parts operators. J. Comput. Phys., 253:418442, 2013.

[26] K. Mattsson and M. H. Carpenter. Stable and accurate interpolation operators for high-order multi-block finite-difference methods. SIAM J. Sci Comput., 32(4):2298-2320, 2010. 
[27] K. Mattsson, F. Ham, and G. Iaccarino. Stable and accurate wave propagation in discontinuous media. J. Comput. Phys., 227:8753-8767, 2008 .

[28] K. Mattsson and J. Nordström. Summation by parts operators for finite difference approximations of second derivatives. J. Comput. Phys., 199(2):503-540, 2004.

[29] K. Mattsson and F. Parisi. Stable and accurate second-order formulation of the shifted wave equation. Commun. Comput. Phys., 7:103-137, 2010.

[30] K. Mattsson, M. Svärd, M.H. Carpenter, and J. Nordström. High-order accurate computations for unsteady aerodynamics. Computers $\mathscr{E}$ Fluids, 36:636-649, 2006.

[31] K. Mattsson, M. Svärd, and M. Shoeybi. Stable and accurate schemes for the compressible navier-stokes equations. J. Comput. Phys., 227(4):2293-2316, 2008.

[32] Ken Mattsson. Summation by parts operators for finite difference approximations of second-derivatives with variable coefficients. Journal of Scientific Computing, 51:650-682, 2012.

[33] J. Nordström and M. H. Carpenter. Boundary and interface conditions for high-order finite-difference methods applied to the Euler and NavierStokes equations. J. Comput. Phys., 148:341-365, 1999.

[34] J. Nordström and M. H. Carpenter. High-order finite difference methods, multidimensional linear problems, and curvilinear coordinates. $J$. Comput. Phys., 173:149-174, 2001.

[35] J. Nordström and J. Gong. A stable hybrid method for hyperbolic problems. J. Comput. Phys., 212:436-453, 2006.

[36] J. Nordström, K. Mattsson, and R.C. Swanson. Boundary conditions for a divergence free velocity-pressure formulation of the incompressible navier-stokes equations. J. Comput. Phys., 225:874-890, 2007.

[37] T. H. Pulliam and D. S. Chaussee. A diagonal form of an implicit approximate-factorization algorithm. J. Comput. Physics, 39:341-363, 1981.

[38] S. De Rango and D. W. Zingg. High-order aerodynamic computations on multi block grids. AIAA Paper, 2001-2631, 2001. 
[39] B. Sjogreen. High order centered difference methods for the compressible navier-stokes equations. Technical report 01.01, RIACS, NASA Ames Research Center, 2001.

[40] B. Strand. Summation by parts for finite difference approximations for d/dx. J. Comput. Physics, 110:47-67, 1994.

[41] John C. Strikwerda. High-order-accurate schemes for incompressible viscous flow. International Journal for Numerical Methods in Fluids, 24:715-734, 1997.

[42] M. Svärd. On coordinate transformation for summation-by-parts operators. Journal of Scientific Computing, 20(1), 2004.

[43] M. Svärd, M. H. Carpenter, and J. Nordström. A stable high-order finite difference scheme for the compressible Navier-Stokes equations, no-slip wall boundary conditions. J. Comput. Physics, 227:4805-4824, May 2008.

[44] M. Svärd, K. Mattsson, and J. Nordström. Steady-state computations using summation-by-parts operators. Journal of Scientific Computing, 24(1):79-95, July 2005.

[45] M. Svärd and J. Nordström. Stability of finite volume approximations for the laplacian operator on quadrilateral and triangular grids. Applied Numerical Mathematics, 51(1), October 2004.

[46] M. Svärd and J. Nordström. On the order of accuracy for difference approximations of initial-boundary value problems. J. Comput. Physics, 218:333-352, October 2006. 